\title{
CHANNEL ESTIMATION IN MOBILE WIRELESS SYSTEMS
}

\author{
Rashmi Mohan, Sagar S, Thejaswini N, Varsha P, Akshaya Y M and Pramela B \\ Department of Electronics and Communication, \\ East West Institute of Technology, Bangalore, India
}

\begin{abstract}
ABTRACT
One of vital parts of the mobile wireless channel is channel estimation significantly improve the performance of the system, especially for $4 G L T E$ and $5 G$ systems. The main goal of this project is to test the time-variant channel estimation using F-OFDM model. It investigates the Mean Square Error (MSE), the Least Squares (LS) method and compares performance of OFDM and F-OFDM using BER vs SNR.
\end{abstract}

Keywords: 4G LTE, 5G, OFDM, F-OFDM, MSE, LSE.

Cite this Article: Rashmi Mohan, Sagar S, Thejaswini N, Varsha P, Akshaya Y M and Pramela B, Channel Estimation in Mobile Wireless Systems, International Journal of Electronics and Communication Engineering and Technology, 10(3), 2019, pp. 01-07. http://www.iaeme.com/IJECET/issues.asp?JType=IJECET\&VType=10\&IType $=3$

\section{INTRODUCTION}

The increasing demands require wireless transmission systems that can support a large number of data hungry users that may be densely located and/or travelling at high velocities. In addition, it is very likely that future wireless systems need to support not only the traditional devices that are directly controlled by a user such as a mobile phone or a computer, but also more or less autonomous devices that communicate amongst each other, the so-called Internet-of-Things (IoT), causing the number of user equipment to increase.

Channel estimation is an important technique especially in mobile wireless network systems where the wireless channel changes over time, usually caused by transmitter and/or receiver being in motion at vehicular speed. Mobile wireless communication is adversely affected by the multipath interference resulting from reflections from surroundings, such as hills, buildings and other obstacles. In order to provide reliability and high data rates at the receiver, the system needs an accurate estimate of the time-varying channel. Furthermore, mobile wireless systems are one of the main technologies which used to provide services such as data communication, voice, and video with quality of service (QOS) for both mobile users and nomadic. The knowledge of the impulse response of mobile wireless propagation channels in the estimator is an aid in acquiring important information for testing, designing or planning wireless communication systems. 
In 4G LTE networks, orthogonal frequency division multiplexing (OFDM) has served as an elegant solution to combat the frequency selectivity and to boost the spectrum efficiency. Recently, it is becoming a consensus that the basic waveform of $5 \mathrm{G}$ should be able to offer including but not limited to: 1 . Tailored services to different needs and channel characteristics, 2. Reduced out-of-band emission (OOBE), 3. Extra tolerance to time-frequency misalignment.

To avoid the limitations of OFDM and to meet the new challenges faced by $5 \mathrm{G}$ waveform, here, in this paper, we present a new enabler for flexible waveform, named as filtered-OFDM (f-OFDM). With subband-based splitting and filtering, independent OFDM systems (and possibly other waveforms) are closely contained in the assigned bandwidth. In this way, fOFDM is capable of overcoming the drawbacks of OFDM whilst retaining the advantages of it.

\section{DESIGN METHODOLOGY OF OFDM AND F-OFDM}

\subsection{ORTHOGONAL FREQUENCY DIVISION MULTIPLEXING (OFDM)}

Orthogonal frequency division multiplexing can accommodate high data rate in the mobile wireless systems in order to handle multimedia services. It is important to understand the OFDM technology because the channel estimation is an integral part of OFDM system. OFDM technology can be used effectively to avoid the effect of frequency-selective fading and narrowband interference from parallel closely spaced frequencies in mobile networks. If there is no orthogonality in the channel, inter-channel interference (ICI) can be experienced. With these vital advantages, OFDM technology has been widely used by many wireless standards such as WLAN, WMAN, and DVB. In OFDM scheme, complex filters are not required and time-spreading can be used without any complications in OFDM.

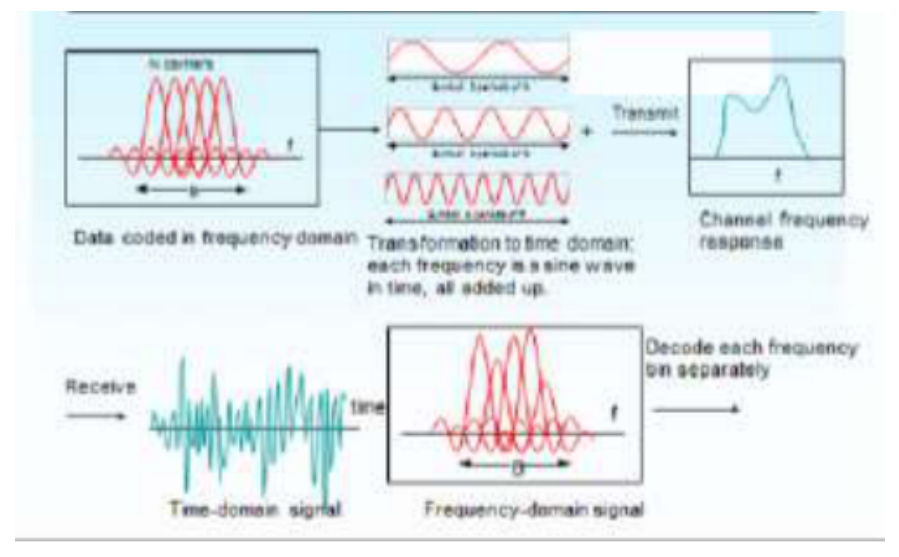

Figure 2.1 OFDM in mobile wireless system

OFDM scheme can also help to manage the single frequency networks (SFN) by sending the same signals at the same frequency using adjacent transmitters without interfering each other. OFDM has been popularly used for various wireless communication systems such as fourth generation $4 \mathrm{G}$ wireless system.

The technology is intended for downlink in the physical layer. The OFDM channel is divided into several narrowband sub-carriers and designed to be orthogonal with each other in the frequency domain for carrying data symbols. Each sub-carrier has parallel narrow bandpass channels at low symbol rate. The advantage of using a low symbol rate is that it is possible to use a guard interval between symbols and to avoid the inter-symbol interference. In the OFDM the total data rate for sub-carriers are equivalent to the single carrier modulation with the same bandwidth. The OFDM plays an important role in the wideband of mobile wireless systems. 


\subsection{FILTERED- ORTHOGONAL FREQUENCY DIVISION MULTIPLEXING (F-OFDM)}

It is a well-known fact that for higher data rates, higher Bandwidth is required. The fifth generation of mobile communication would have higher bandwidth to fulfil this need. FilteredOFDM would utilize this bandwidth in such way that the entire spectrum is divided into several smaller sub-bands. Each of these sub-bands would contain a personalized waveform in order to satisfy the requirements of different services offered by the network. This means that parameters like cyclic prefix (CP) transmit time interval (TTI) and pacing between the subcarriers in each sub-band would be in accordance with the nature of service. After this, each of these sub-bands would be passed through a tailored filter in order to reduce interference among each other. On the other hand, to support asynchronous transmission across sub-bands and rule out the need of global synchronization, the time domain-based orthogonality between the subbands is fragmented purposefully. Consequently, F-OFDM would also reduce the spectral consumption of the guard band, which would increase the spectral efficiency of the system.

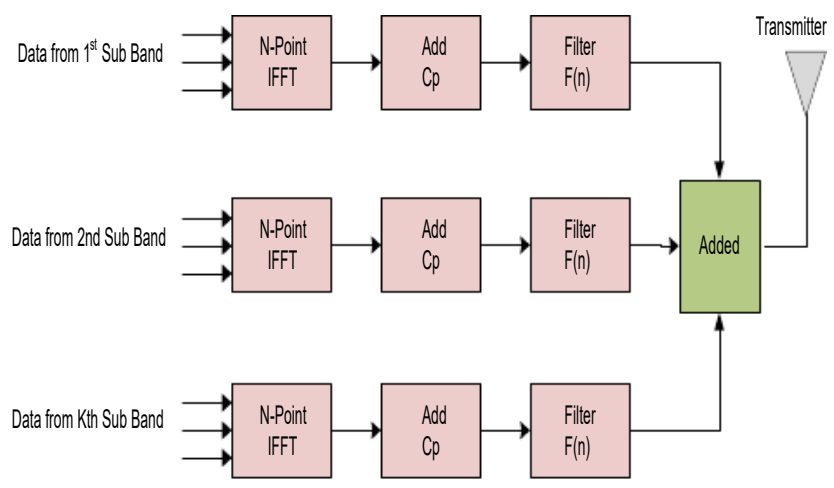

Figure 2.2 F-OFDM Transmitter

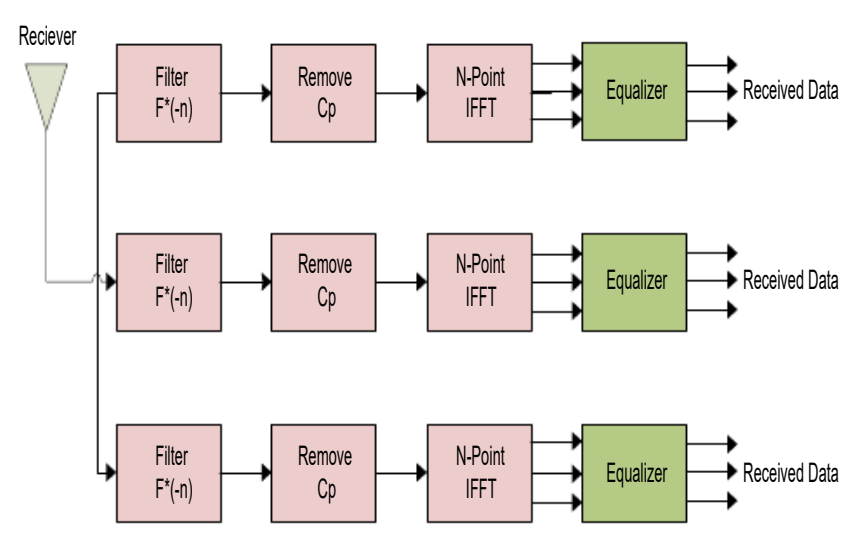

Figure 2.3 F-OFDM Receiver

Figure 2.2 and Figure 2.3 show the basic transmitter and receiver structural design for FOFDM respectively. The primary advantage of F-OFDM is that the space between the subcarriers in each of the sub-band can be different. It has been mentioned earlier that the each of these sub-bands, depending upon the services, can have different parameters.

It is worth mentioning that the sub-bands do not overlap each other and instead of a guard band, a guard tone is added between them. This guard tone is smaller than that of the guard band used in CP-OFDM and only utilizes $2 \%$ of the allocated spectrum which in the case of CP-OFDM was $10 \%$. 


\subsection{Operations of Transmitter and Receiver of F-OFDM}

- At the transmitter side, the signals[n] obtained after N-point IFFT and CP insertion is then passed through a designed spectrum shaping filter $\mathrm{f}[\mathrm{n}]$, i.e., the transmitted signal can be expressed as

$$
\mathrm{s}[\mathrm{n}]=\mathrm{s}[\mathrm{n}] * \mathrm{f}[\mathrm{n}] .
$$

- The receive module has inverse operations as transmit module. The received signal is firstly passed through the filter $\mathrm{f} *[-\mathrm{n}]$ which is matched to the transmitter filter . Then the signal of F-OFDM at receiver is processed as the same as regular OFDM receiver.

- The IFFT/CP insertion block, filtering module is used. In f-OFDM, a filter is applied to time domain OFDM symbol in order to improve out of band radiation of sub-band signal, while at the same time complex domain orthogonality of the OFDM symbols is being maintained.

\section{SOFTWARE IMPLEMENTATION}

An OFDM and F-OFDM system is modeled using Matlab to allow various parameters of the system to be varied and tested. The aim of doing the simulations is to measure the performance of these systems under different channel conditions, and to allow for different configurations to be tested.

\subsection{Flow chart Main Toolbox}

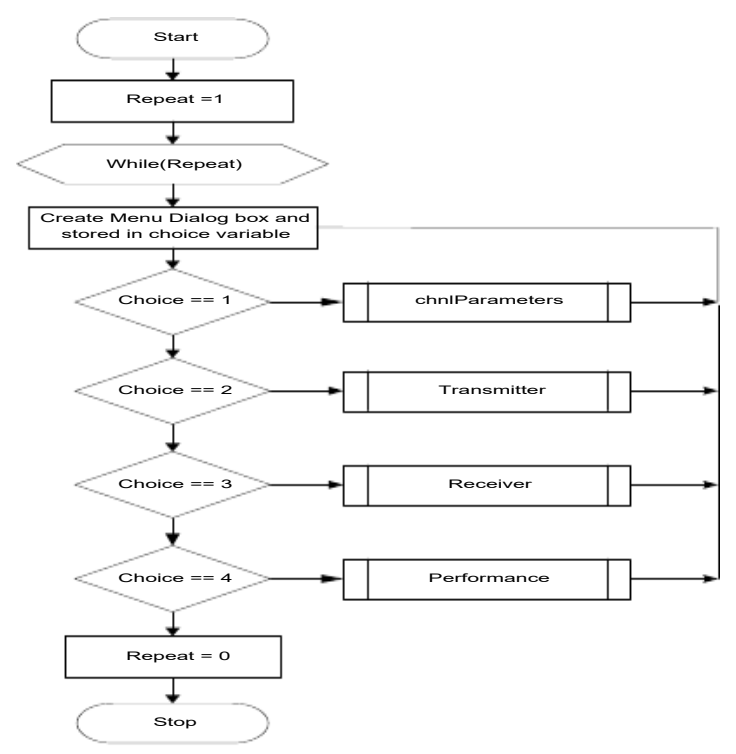

Figure 3.1 Main tool box

Fig 3.1 represents the overall implementation work flow of our proposed method. While implementation the overall work is divided into four choices. Choice 1 initializes the channel parameters only if choices $==1$. Once channel parameters are initialized the choice is switched to choice $=2$ where Transmitter is incorporated to transmit the information bits. Once transmitter bits are implemented it has to send to the receiver so in that case choice is switched to choice $=3$. So during choice $==3$ receiver is implemented to receive the transmitted bits. Once transmission and reception is done successfully than choice is switched to choice $==4$ to determine the performance of bit error rate (transmission and reception bit error rate). 
Details the channel parameters are initialized before transmission which include defining the number of sub carriers and carrier frequency required to transmit information over channel. Specifying the length of transmitted filter and receiving filter. Initialize the OFDM and FOFDM modulation at the transmitter side.

At the transmitter side declare the velocity and power delay for transmitting bits. Assign the transmitted power to the bits. Fast fading refers to variation in bit strength with respect to time. This variation can be result of wireless communication channel between the transmitter and receiver. Map binary data to transmitting symbol and specify Pilot \& Date Locations. Transmitted signal in the time domain using OFDM and FOFDM Modulation which further convolve or multiplies with channel. Calculate the transmitted power over time and power spectral density.

At the transmitter bits are transmitted over a channel while before this transmitted bits receives at the receiver this transmitted bits are multiplied with noise called as channel noise. So the received bits are the combination of noise and transmitted bits. Once transmitted information bits are received it is demodulated using OFDM and FOFDM. For channel estimation extract the received pilots and determine the least square and mean square estimation. Perform interpolation to estimation of a value within two known values in a sequence of values. Quantize the received symbols and perform de-mapping to bits. Finally calculate the BER.

\section{RESULT}

The result of our simulation with the different parameters for the F-OFDM systems are shown in the below graph using MSE and LSE.

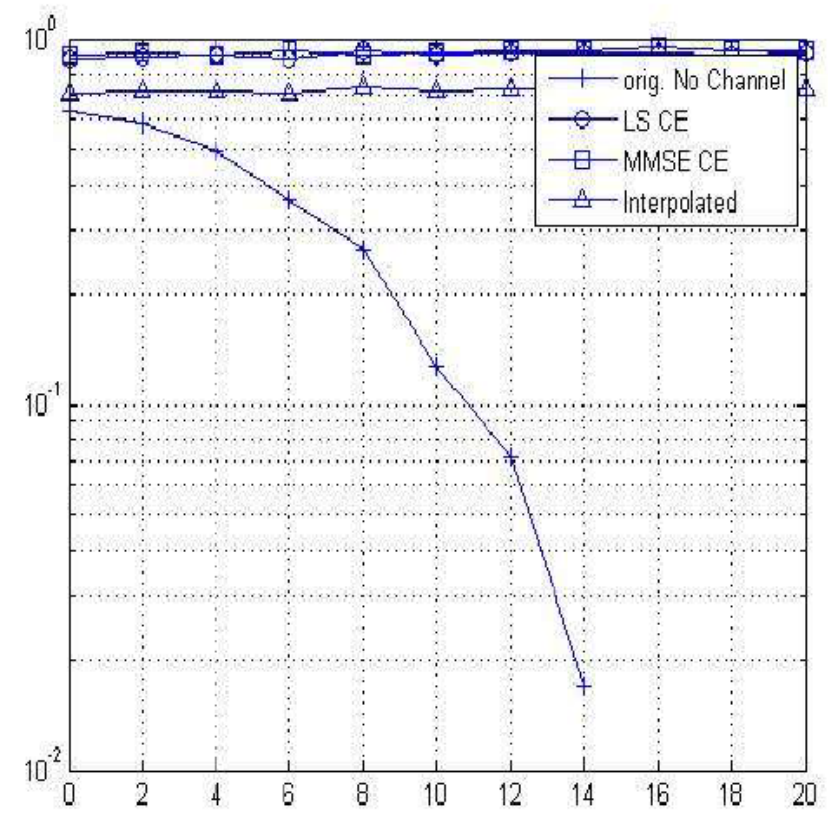

Figure 4.1 Interpolated, MSE and LSE signal

The performance of the OFDM and F-OFDM is compare using BER vs SNR. The obtained graph is as shown. 


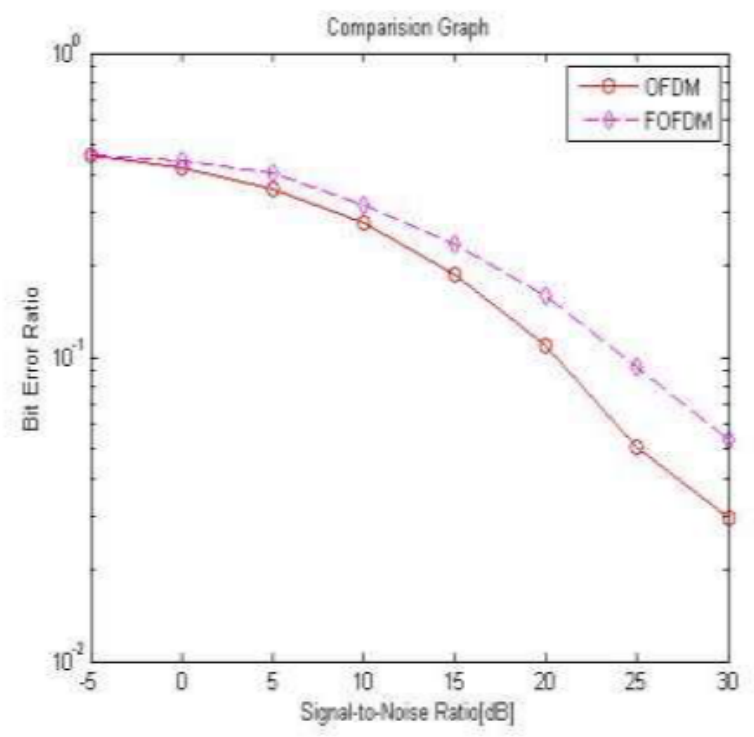

Figure 4.2 Comparison of performance of OFDM vs F-OFDM

The following table shows the values of Bit Error Ratio to the respective Signal to Noise Ratio:

Table. Comparison values of performance of OFDM and

\begin{tabular}{|c|c|c|c|c|c|c|}
\hline SNR (dB) & $\mathbf{- 5}$ & $\mathbf{0}$ & $\mathbf{5}$ & $\mathbf{1 0}$ & $\mathbf{2 0}$ & $\mathbf{3 0}$ \\
\hline OFDM & 0.4236 & 0.3616 & 0.2743 & 0.1915 & 0.0397 & 0.0047 \\
\hline F-OFDM & 0.4744 & 0.4311 & 0.4056 & 0.3422 & 0.1361 & 0.0155 \\
\hline
\end{tabular}

F-OFDM using BER vs SNR

\section{CONCLUSION}

In this work, we have studied LSE and MMSE estimators for both block type and comb type pilot arrangement. The estimators in this study can be used to efficiently estimate the channel in an OFDM and F-OFDM systemS given a certain knowledge about channel statistics. For high SNRs the LSE estimator is both simple and adequate. The MMSE estimator has good performance but high complexity. The LSE estimator has low complexity, but its performance is not as good as that MMSE estimator basically at low SNRs. We also used interpolation techniques for channel estimation.

\section{REFERENCES}

[1] Xiaojie Wang, "Channel Estimation and Equalization For 5G Wireless Communication Systems", IEEE 2014.

[2] Xi Zhang, Ming Jia, LeiChen, Networks, IEEE Globecom, San Diego, CA, Dec. 2015 2015. Jianglei Ma, JingQiu, Filtered-OFDM-Enabler for Flexible Waveform in the $5^{\text {th }}$ Generation Cellular

[3] QiweiZheng, FanggangWang, Xia Chen, Yinsheng Liu, DeshanMiao, and Zhuyan Zhao, Comparison of 5G Waveform Candidates in High Speed Scenario, IEEE $32^{\text {nd }}$ URSI GASS, Montreal, 19-26 August 2017.

[4] Sami Valkonen, Comparison of Fast-Convolution Based Filtered OFDM And Windowed OFDM for 5G Physical Layer Publication, IEEE 2017. 
[5] Swati Mishra, Mohammad Umar, Shalini Kashyap, Performance Analysis of OFDM for 5G MIMO System, International Research Journal of Engineering and Technology (IRJET), Volume 04, Issue 05, May-2017.

[6] Naveena Koppati, Pavani, Dinesh Sharma, Purnima K Sharma, MIMO to LSMIMO: A Road to Realization of 5G, 2017

[7] Syed Shoaib UllahShah, Majid Ashraf, RuhulAmin Khalil, TariqullahJan, M. Inayatullah Babar, "Implementing Enhanced MIMO with F-OFDM To Increase System Efficiency for Future 5G Cellular Networks" International Journal of Communication Networks and Information Security (IJCNIS) Vol. 10, No. 2, August 2018.

[8] Alaa Ghaith, Filtered Orthogonal Frequency Division Multiplexing: A Waveform Candidate For 5G" IEEE American Journal of Engineering Research (AJER), Volume-7, Issue-1, pp-99-107, 2018.

[9] Coleri, S, Ergen, M., Puri, A. and Bahai, A., "Channel Estimation Techniques based on Pilot Arrangement in OFDM systems," IEEE Transaction on Broadcasting (September 2002). 